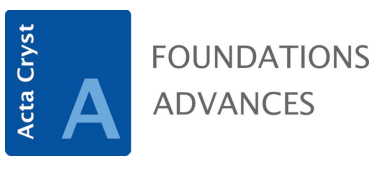

ISSN 2053-2733
Keywords: book review; crystal symmetry; crystal physics.

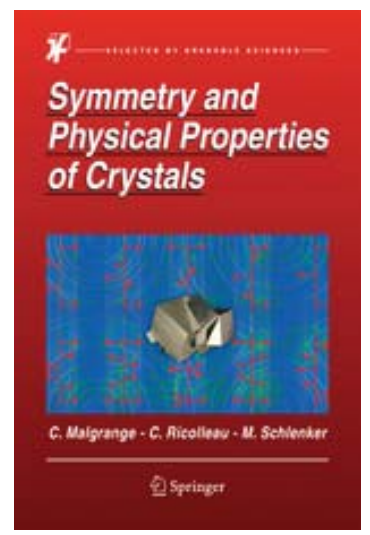

(C) 2017 International Union of Crystallography

\section{Symmetry and Physical Properties of Crystals. By Cécile Malgrange, Christian Ricolleau and Michel Schlenker. Springer, 2014. Pp. xxv + 522. Price EUR 94.94, GBP 81.00, USD 119.99. ISBN 978-94-017-8992-9.}

\author{
Hans Grimmer*
}

Research with Neutrons and Muons, Paul Scherrer Institut, WLGA/019, CH-5232 Villigen PSI, Switzerland. *Correspondence e-mail: hans.grimmer@psi.ch

This book is aimed at undergraduate and graduate students in materials science and materials-related aspects of electrical and optical engineering, and at researchers investigating or applying elastic, electric and optical properties of crystals.

After an introduction to crystalline matter in Chapter 1, Chapters 2-7 deal with the point-group (macroscopic) and space-group (microscopic) symmetry of crystals. In Chapter 3 on crystal lattices, reciprocal lattices and surface crystal lattices are also discussed. Chapter 8 gives an introduction to chemical bonds in crystals and Chapters 919 deal with crystal physics, discussing physical properties that can be described by tensors. Tensors are introduced in Chapters $9-10$ and used to discuss the direction dependence of elastic properties in Chapters 11-14; Chapter 15 investigates the interaction between thermal, electric and elastic properties while Chapters 16-19 are devoted to optical properties of crystals. Numerous exercises allow the reader to gain a deeper understanding. Detailed solutions of the exercises are given on the 72 pages of Chapter 20.

The phenomena discussed are clearly defined and their direction dependence carefully described. Many chapters start with an introduction, where results on which the chapter is based are summarized; also relevant previously derived results are recalled. This helps understanding even if one does not remember all the results presented in previous chapters. To liven up the text, complementary material has been added to eight chapters dealing qualitatively with crystallographic topics not treated in detail in the book. They describe crystal growth, structure determination, magnetic structures, plastic deformation and crystal defects, electro- and magnetostriction, magnetic optical rotation, and secondharmonic generation. I enjoyed reading these as a welcome change to the detailed mathematical derivations in the main text.

Let me compare Chapters 9-19 with the contents of Nye's classic text Physical Properties of Crystals (Nye, 1985). Both texts say little about magnetic structures and phenomena; they are mentioned in the text under review in three of the items of complementary material mentioned above. Transport phenomena are dealt with only by Nye, elastic waves and light polarization only in the present text. Both texts are exceptionally clearly written. Whereas Nye concentrates on describing the physical phenomena, the present text also sketches practical applications.

My main criticism concerns the confusion of 'crystal systems' and 'lattice systems'. The space groups belonging to the hexagonal crystal family can be split into two lattice systems according to their lattice (rhombohedral or hexagonal) or, alternatively, into two crystal systems according to their point group (trigonal or hexagonal). These terms are defined in Volume A of International Tables for Crystallography (see Wondratschek, 2002 or Souvignier, 2016). To give an example: I find it irritating to read in Table 12.1 that quartz at room temperature belongs to the rhombohedral crystal system; quartz has a hexagonal lattice and a trigonal point group.

The last sentence of Section 5.2.3 should be replaced by: We can note that the order of the groups $\bar{n}$ is equal to $n$ if $n$ is even, equal to $2 n$ if $n$ is odd.

The word 'direct' is used in several different senses, for which more common, specific notations exist: direct trihedron $\mathbf{a}, \mathbf{b}, \mathbf{c} \rightarrow$ right-handed basis $\mathbf{a}, \mathbf{b}, \mathbf{c}$; direct permutation $i j k \rightarrow$ even permutation $i j k$; direct sense $\rightarrow$ counter-clockwise sense; direct 


\section{book reviews}

trirectangular trihedron $\rightarrow$ right-handed orthogonal basis. The notations 'motif', 'lozenge' and 'arseniate' are used instead of the more common 'pattern', 'rhombus' and 'arsenate', respectively.

I noted a small number of typos in formulas and the text.

The book provides a sound basis for students and researchers to investigate and apply the physical phenomena treated in the text. It can be highly recommended.

\section{References}

Nye, J. F. (1985). Physical Properties of Crystals, 2nd ed. Oxford: Clarendon Press.

Souvignier, B. (2016). International Tables for Crystallography, Vol. A, Space-Group Symmetry, 6th ed., ch. 1.3, edited by M. I. Aroyo. Chichester: Wiley.

Wondratschek, H. (2002). International Tables for Crystallography, Vol. A, Space-Group Symmetry, 5th ed., ch. 8.2, edited by Th. Hahn. Kluwer: Dordrecht. 